\title{
Analyzing Behavioral Markers of Autistic Children Using Eye Tracking Data
}

\section{Seyedeh Negin Seyed Fakhari, Foad Ghaderi*}

Human Computer Interaction Lab, Faculty of Electrical and Computer Engineering, Tarbiat Modares University, Tehran, Iran

\section{A BSTRACT}

Introduction: Autism Spectrum Disorder (ASD) is a neurodevelopmental disorder that occurs in the early years of life and is characterized by social impairment, verbal and non-verbal communication difficulties as well as stereotypical behaviors. Rehabilitating autistic children at the early stages of growth, in which their brain is highly flexible, yields to enhanced treatment process and provides the chance of utilizing their talents. In other words, late detection and treatment will leave these children's behavior unchanged until adulthood. Considering the role of eyes, as one of the most valuable sources of information in social interactions and the different patterns of eye behaviors in autistic children in response to social stimuli, the non-invasive eye tracking technique is an appropriate approach to early diagnosis of this disorder. This way it is possible to investigate how visual stimuli are processed in autistic people at different ages. Conclusion: This study is a review of the previous studies in the field of eye-tracking data analytics conducted with the aim of identifying the autistic and normal children eye movement patterns in response to social stimuli. The results of published investigations confirm that eye tracking is an effective approach for identifying the different patterns of eye movements in autistic children compared to normal subjects. These differences can be assumed as the basis for developing intelligent ASD screening systems.

\section{Key words:}

1. Autism Spectrum Disorder

2. Early Diagnosis

3. Eye Movements

4. Intelligence

*Corresponding Author: Foad Ghaderi

E-mail:fghaderi@modares.ac.ir 


\section{تحليل نشانغرهاى رفتارى كودكان مبتلا به اتيسم با بهرهيرى از دادههاى رديابى جشم}

\section{سيده نخين سيد فخارى، فؤاد قادرى"}

آزمايشكاه تعامل انسان و كامييوتر، دانشكده مهندسى برق و كامييوتر، دانشكاه تربيت مدرس، تهران، ايران

\section{اطلاعات مقاله:}

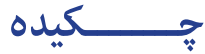

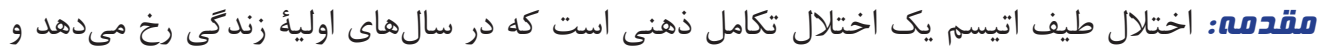

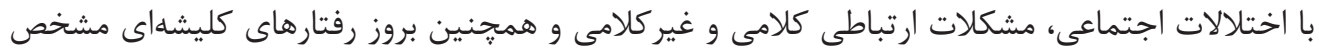

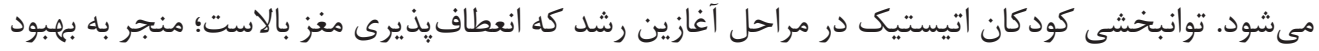

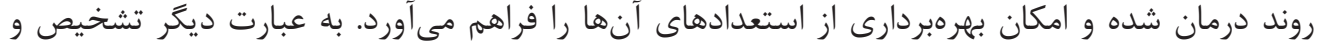

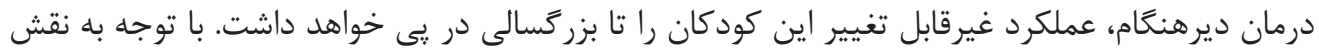

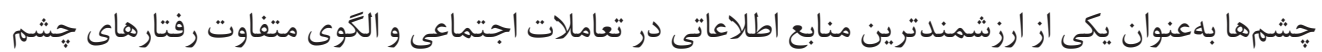

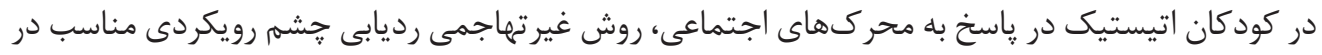

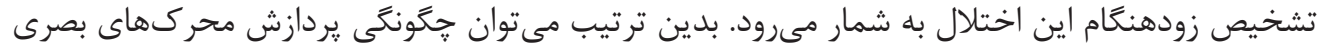

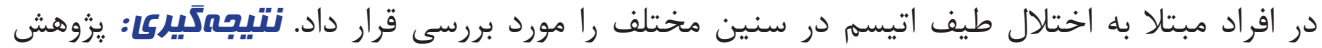

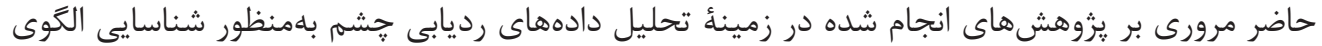

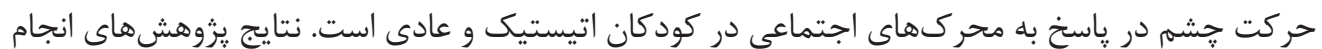

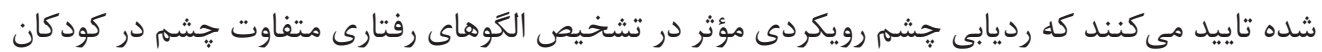

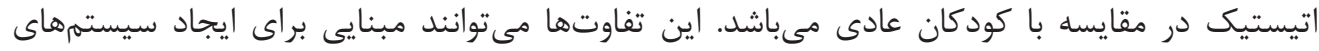

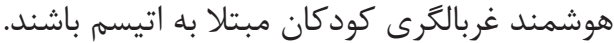

كليد وازهها:

ا. اختلال طيف اتيسم

r.

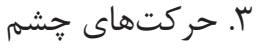

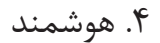

" نويسنده مسئول: فؤاد قادرى

آدرس الكترونيكى: fghaderi@modares.ac.ir 


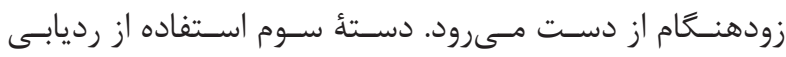

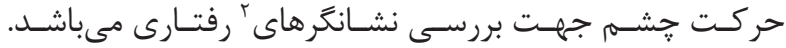

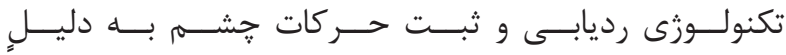

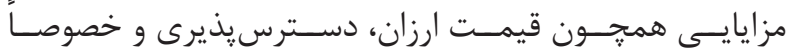

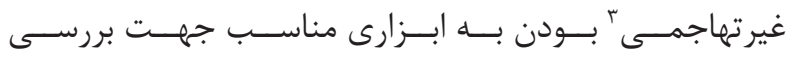

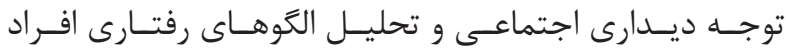

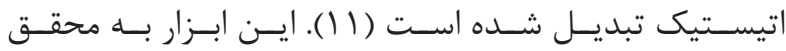

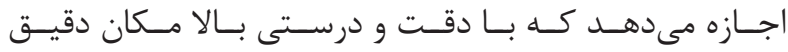

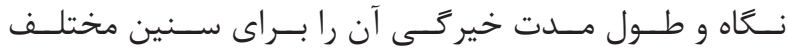

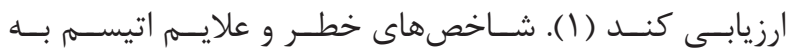

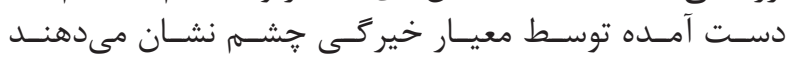

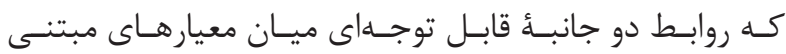

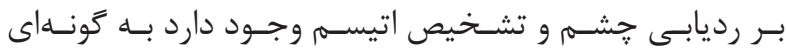

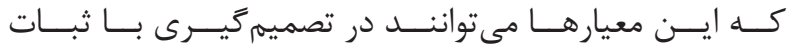

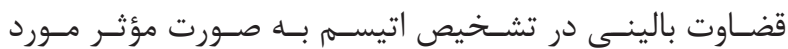

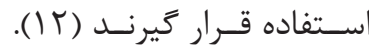

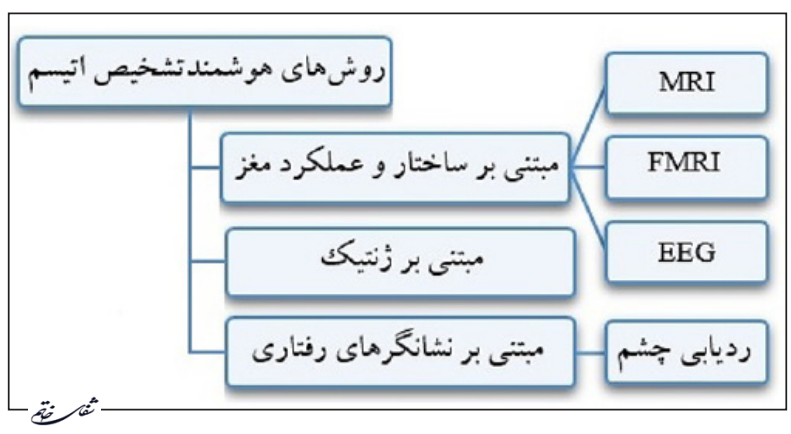

تصوير ا- روشهاى هوشمند تشخيص اتيسم.

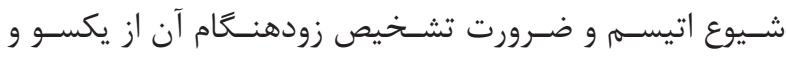

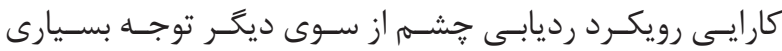

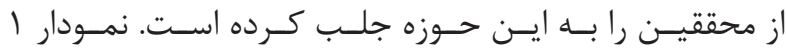

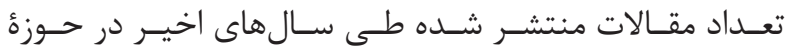

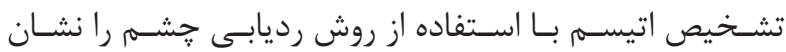

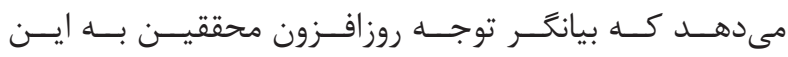

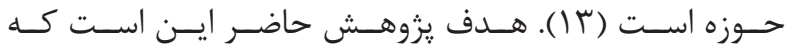

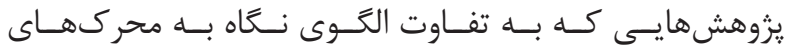

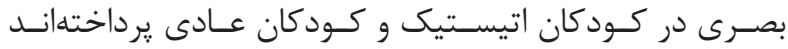

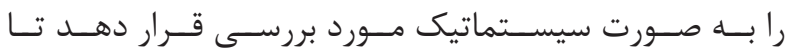

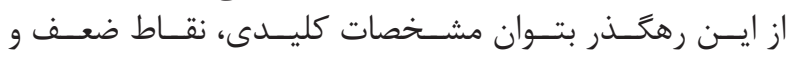

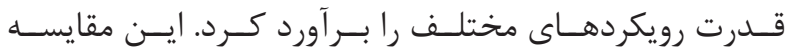

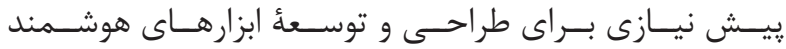

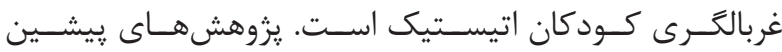

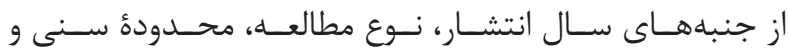

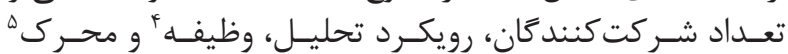

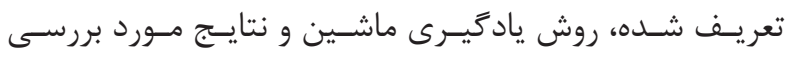

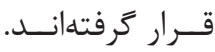

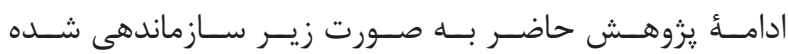

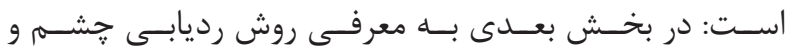

${ }^{1}$ Neurodevelopmental

${ }^{2}$ Biomarker

${ }^{3}$ Noninvasive
مقدمه - - - مقد

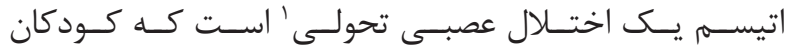

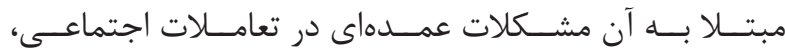

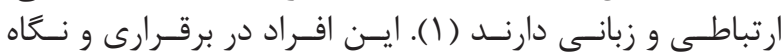

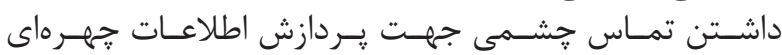

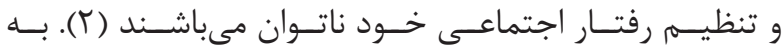

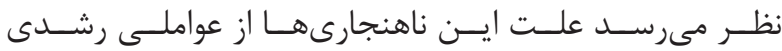

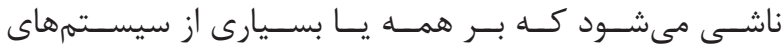

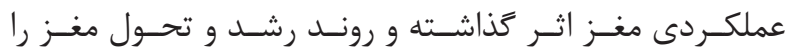

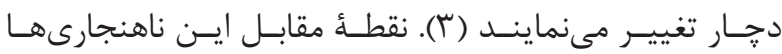

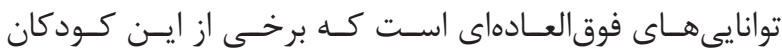

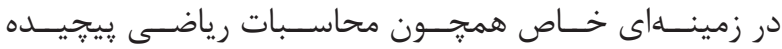

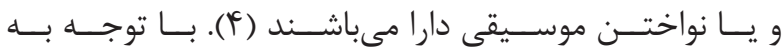

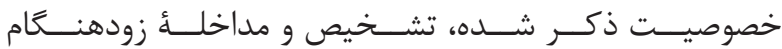

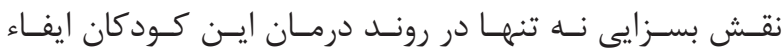

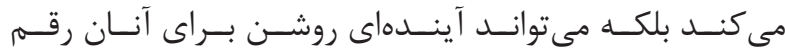

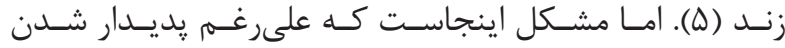

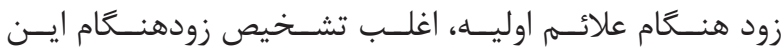

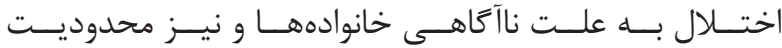

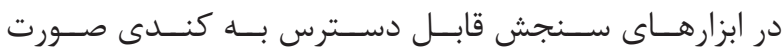

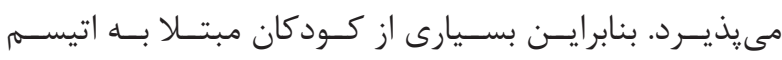

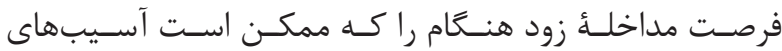

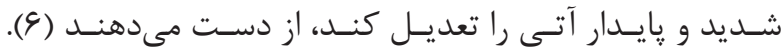

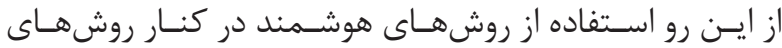

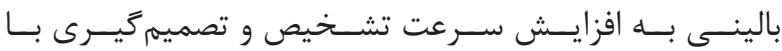

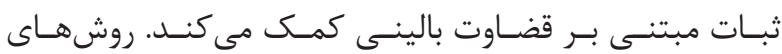

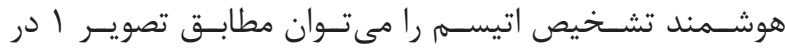

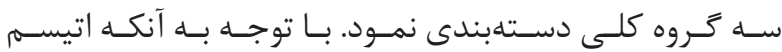

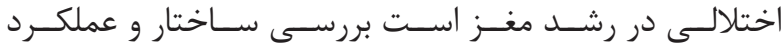

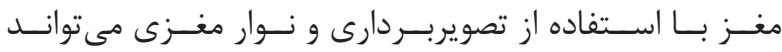

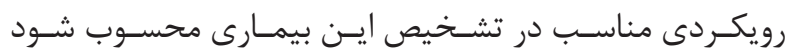
(V، N )

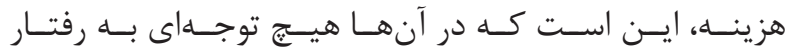

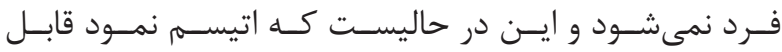

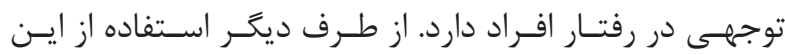

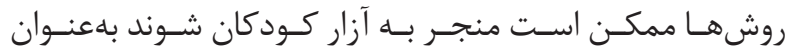

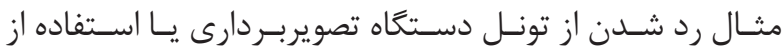

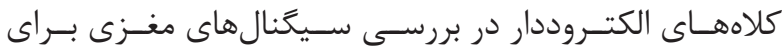

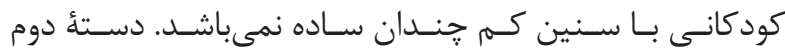

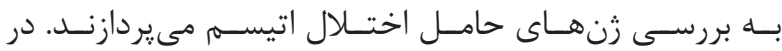

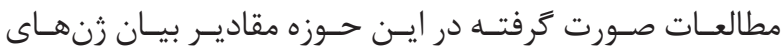

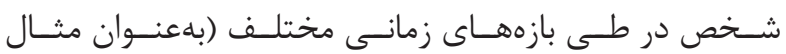

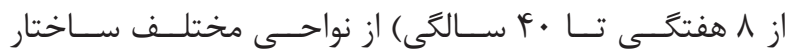

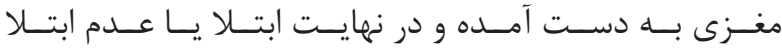

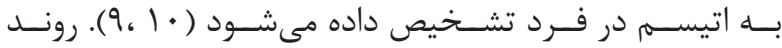

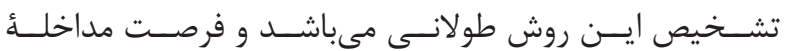

${ }^{4}$ Task

${ }^{5}$ Stimuli 


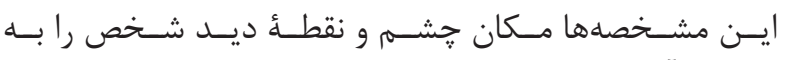

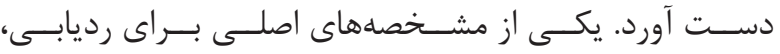

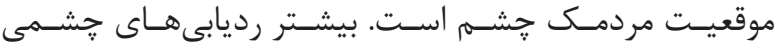

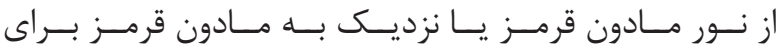

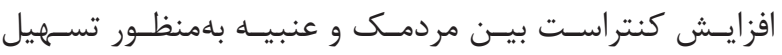

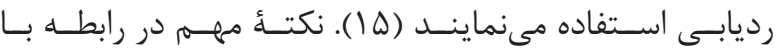

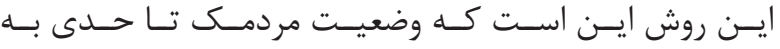

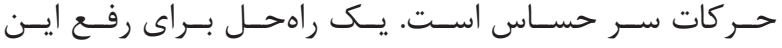

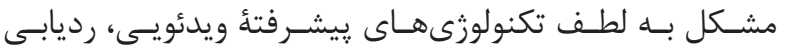

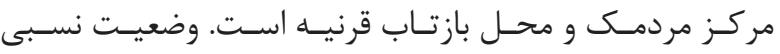

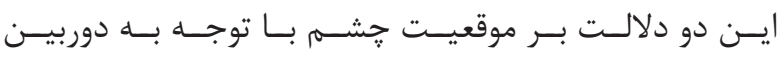

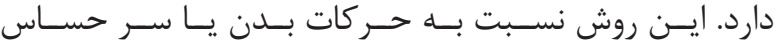

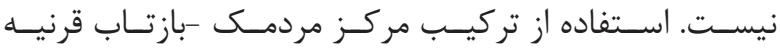

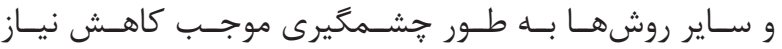

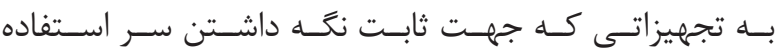

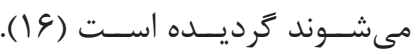

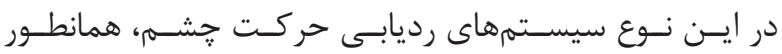

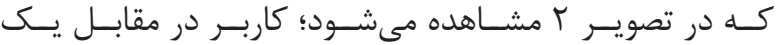

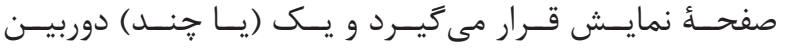

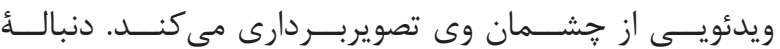

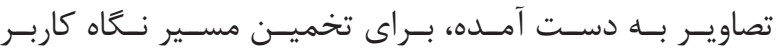

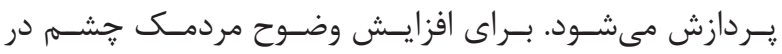

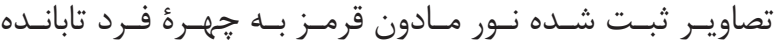

مى شـــود.

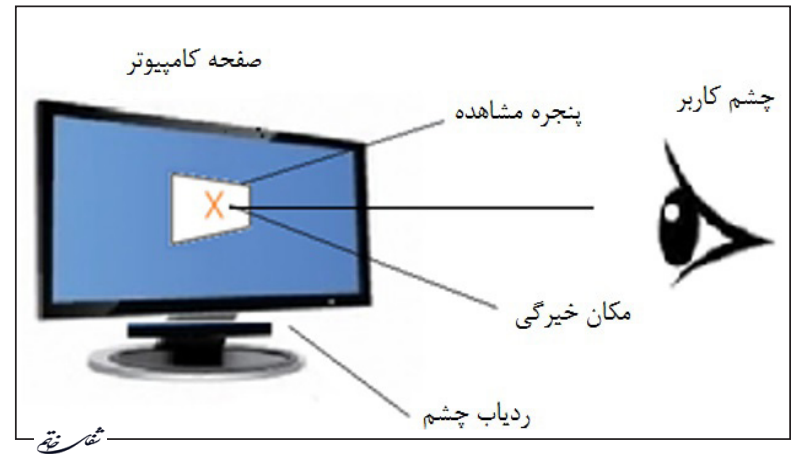

تصوير r- سيستم رديابى جشم.

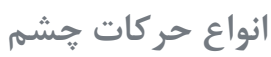

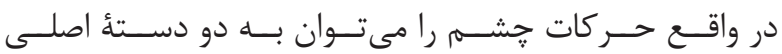

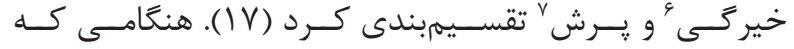

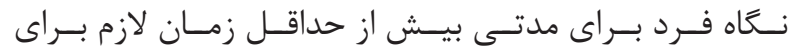

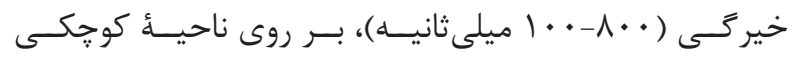

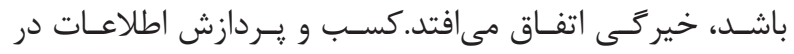

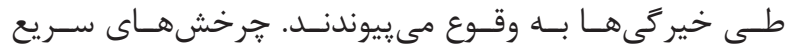

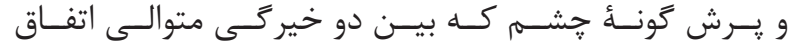

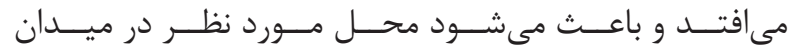

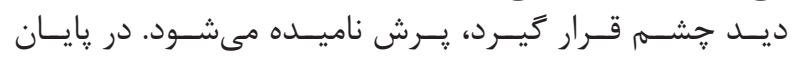

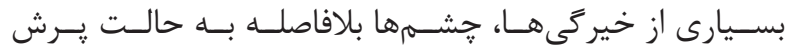

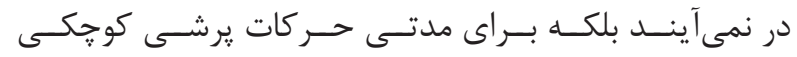

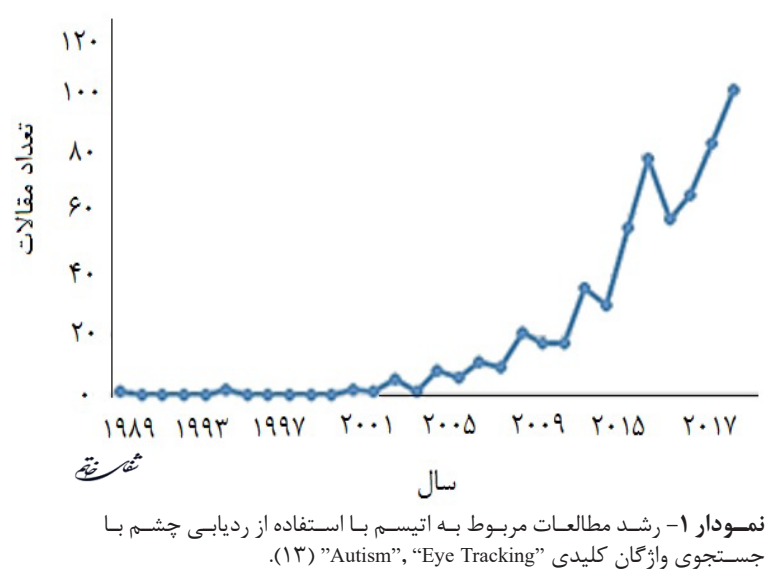

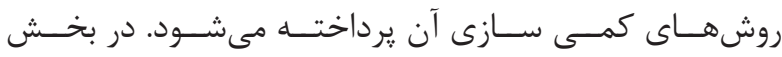

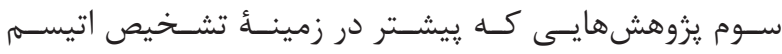

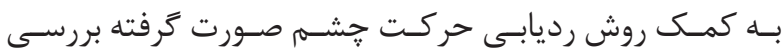

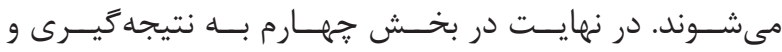

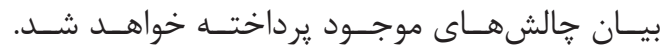

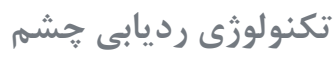

ميـزان توجــه اجتماعـى و ارتباطـات غير كلامسى نشـانكر هايى

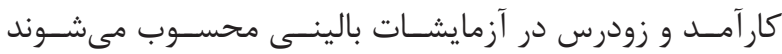

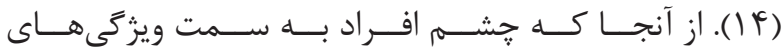

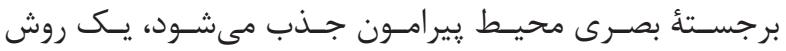

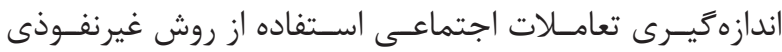

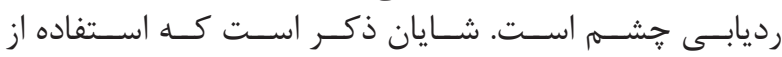

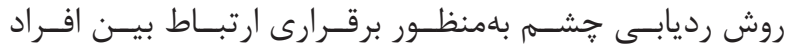

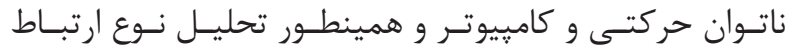

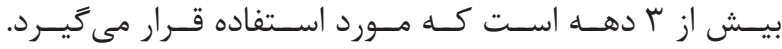

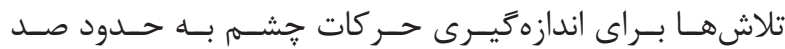

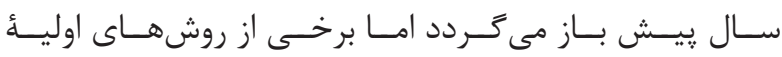

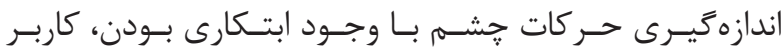

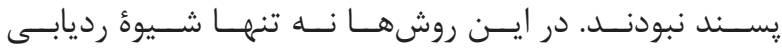

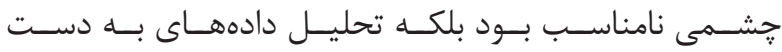

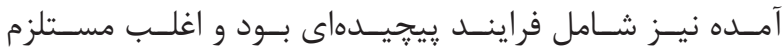

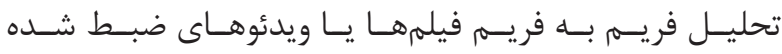

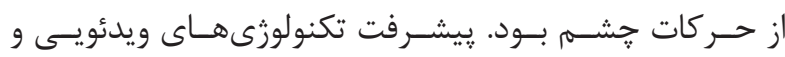

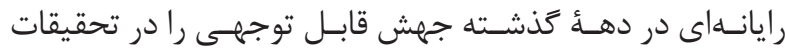

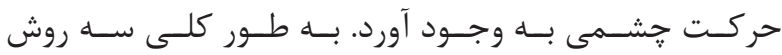

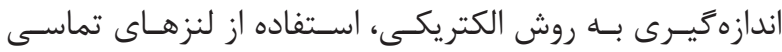

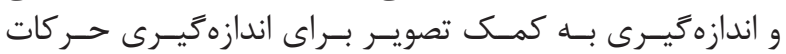

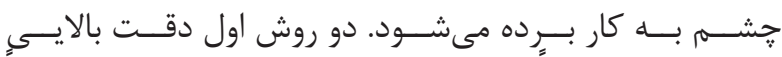

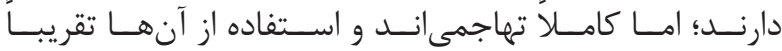

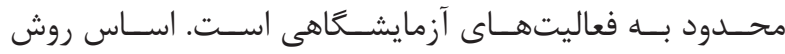

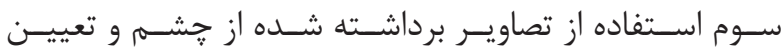

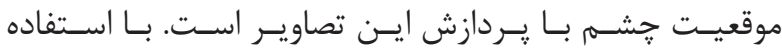

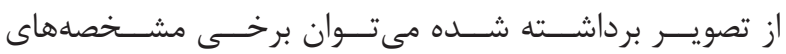

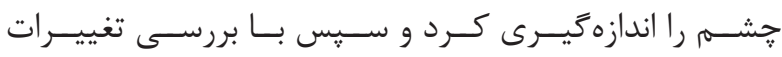




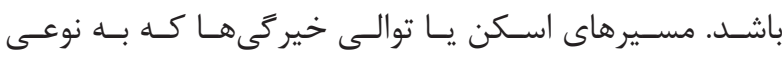

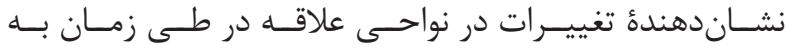

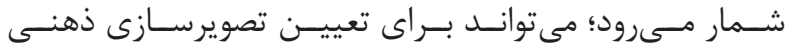

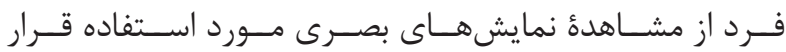

كيــرد (1)

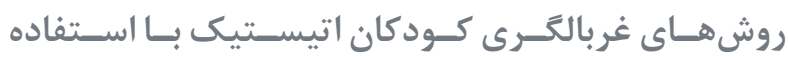

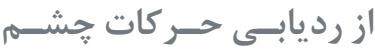

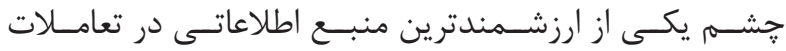

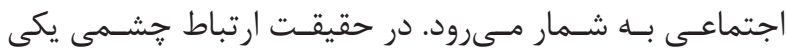

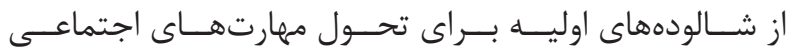

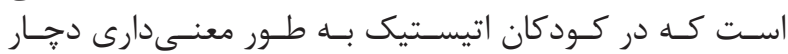

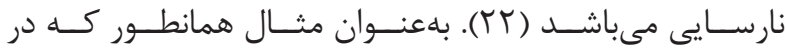

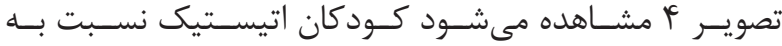

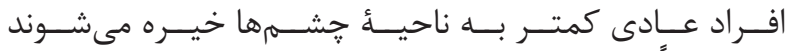

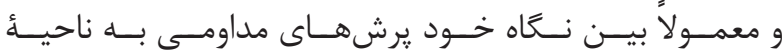

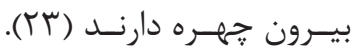

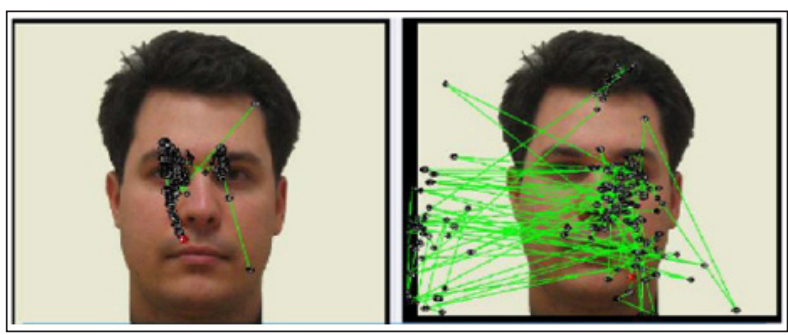

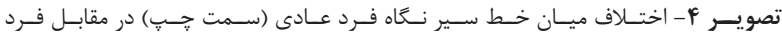

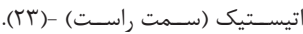

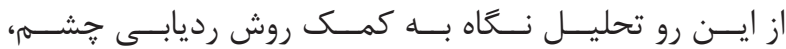

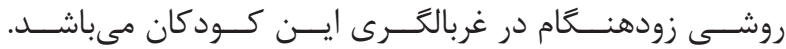

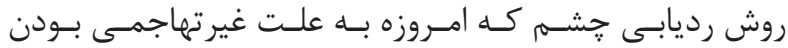

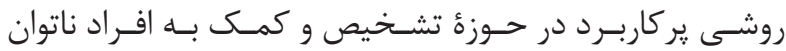

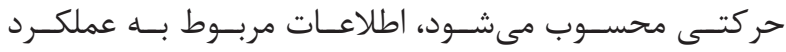

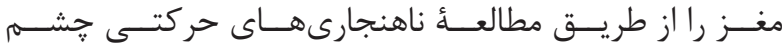

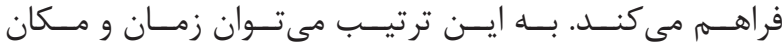

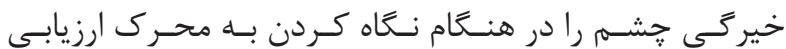

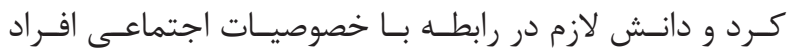

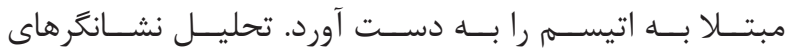

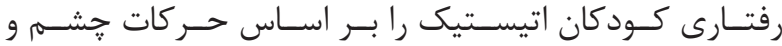

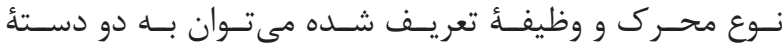

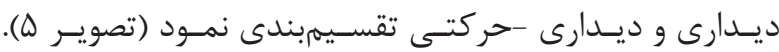

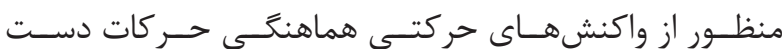

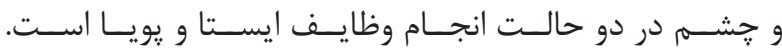

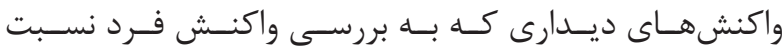

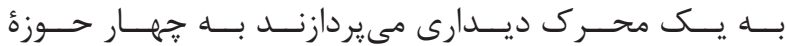

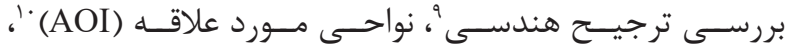

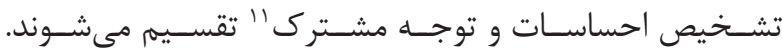

${ }^{8}$ Glissade

${ }^{9}$ Geometric preference

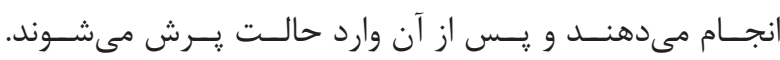

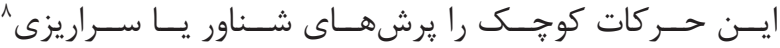

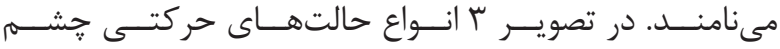

قابـل مشــاهده اسـت (1) (1).

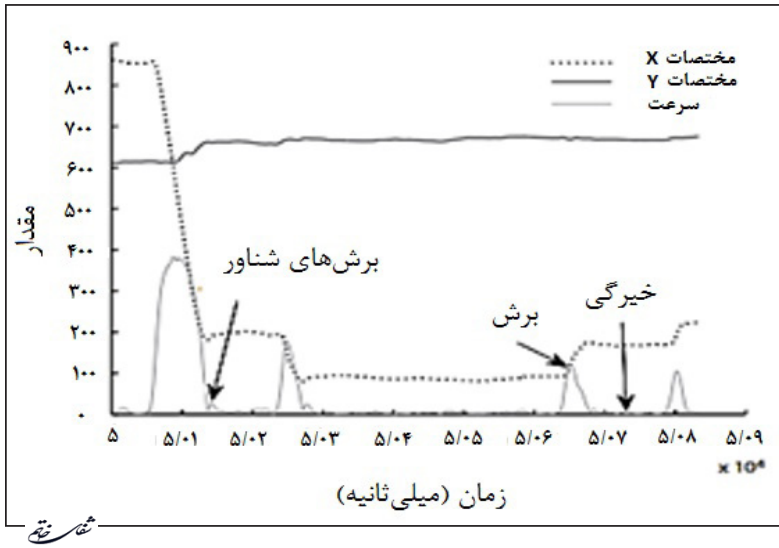

تصوير ب- انواع حالتهاى حركتى خشم (1).). اندازمَيرى حركات جشم

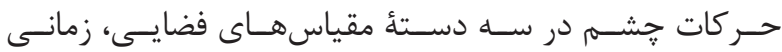

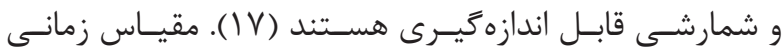

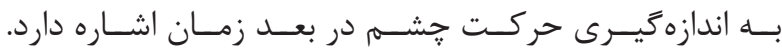

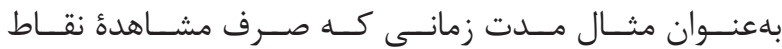

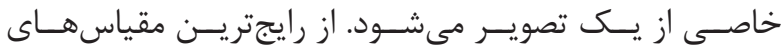

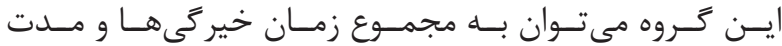

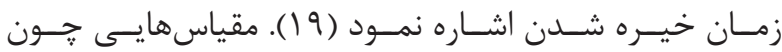

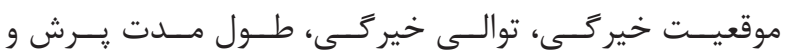

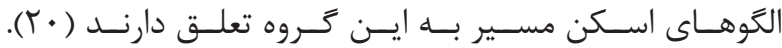

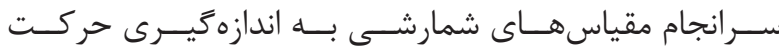

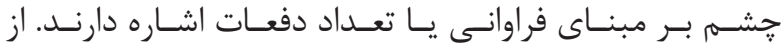

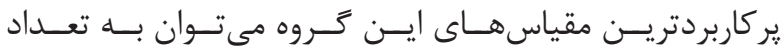

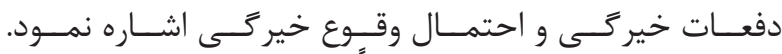

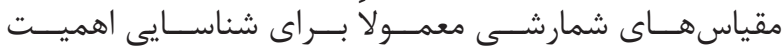

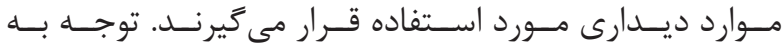

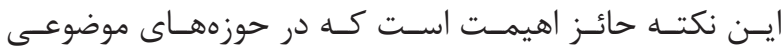

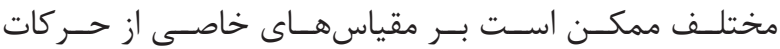

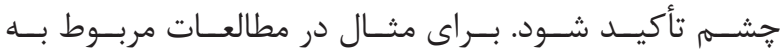

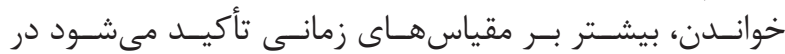

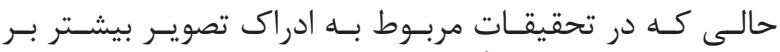

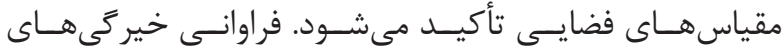

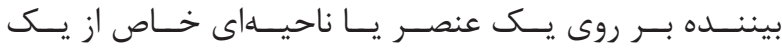

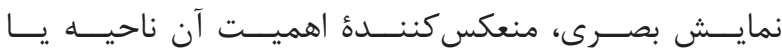

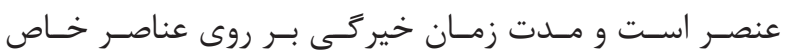

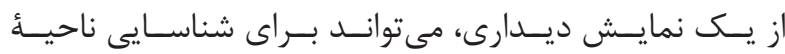

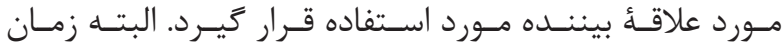

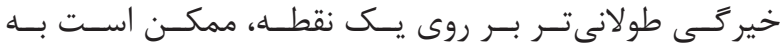

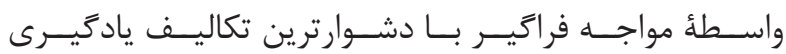

\footnotetext{
${ }^{10}$ Areas-of-interest
}

${ }^{11}$ Joint attention 


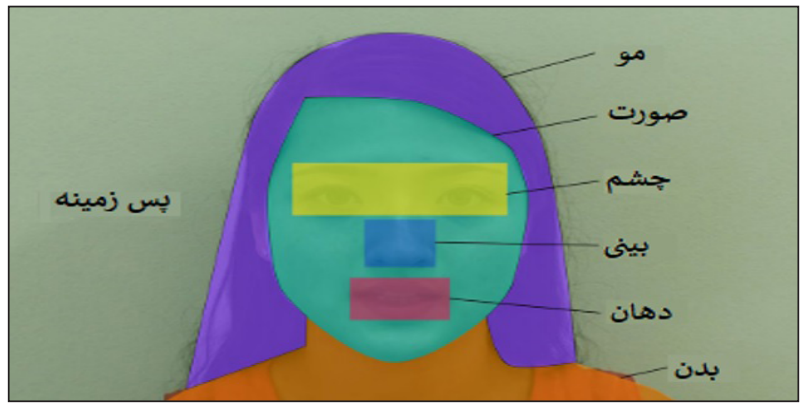

تصوير V- نواحى مورد علاقئ جهره (T)).

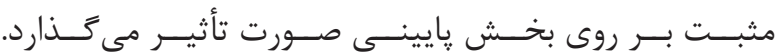

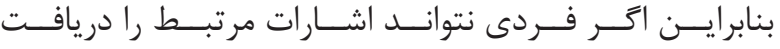

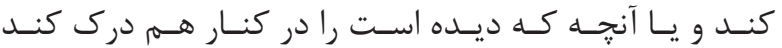

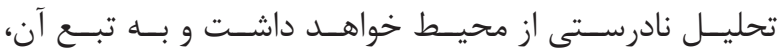

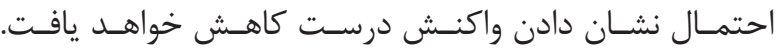

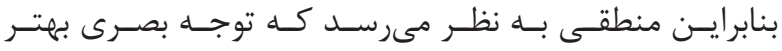

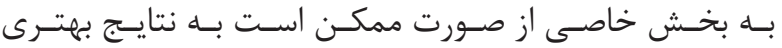

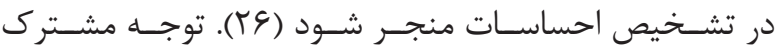

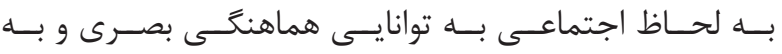

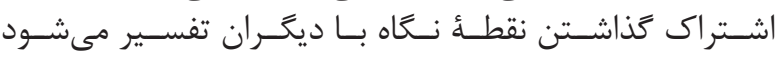
(YV)

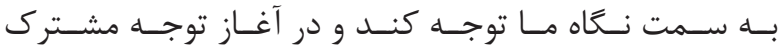

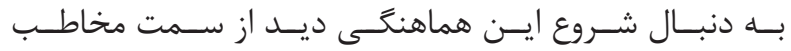

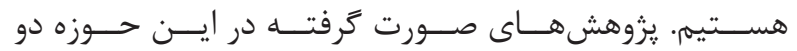

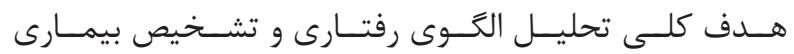

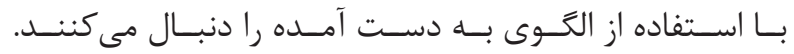

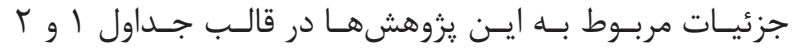

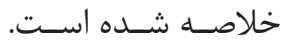

نتايج اين مطالعات نشان مىدهند كه:

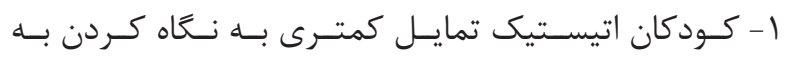

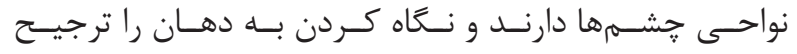

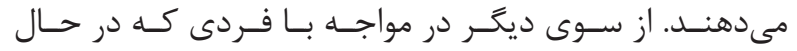

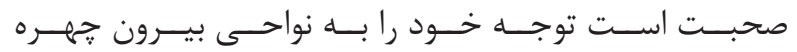

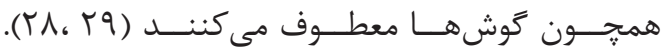

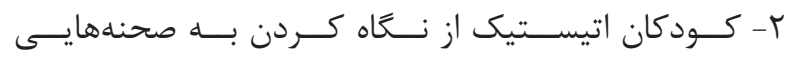

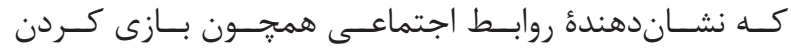

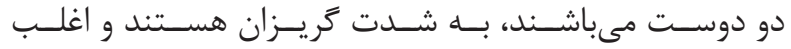

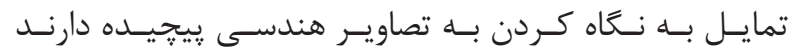

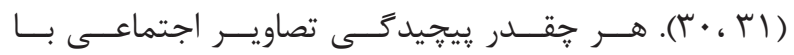

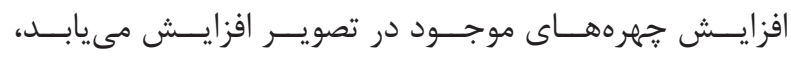

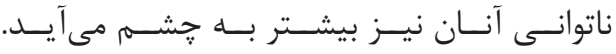

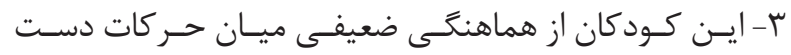

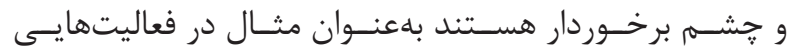

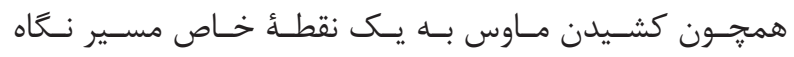

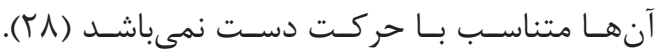

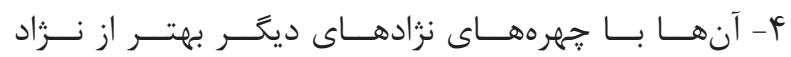

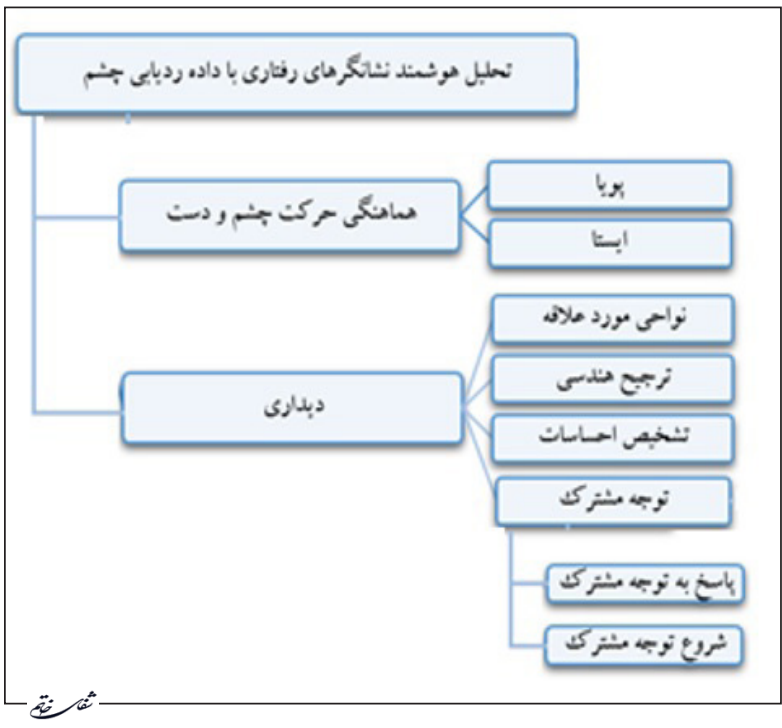

تصويــر هـ - دسـتهبندى تحليـل هوشــمند ارتباطـات غير كلامسى بــا اسـتفاده از رديابـى

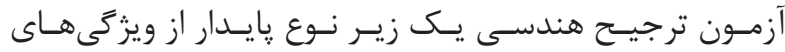

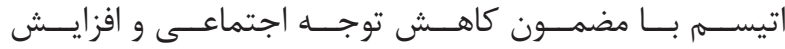

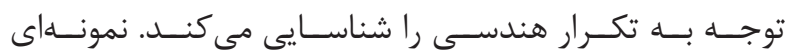

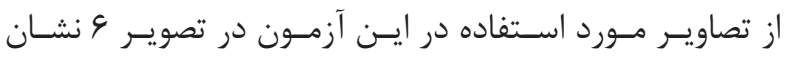

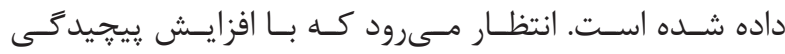

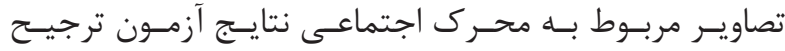

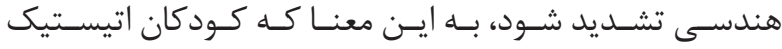

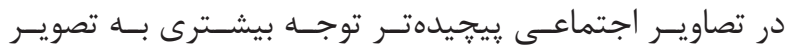

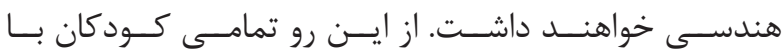

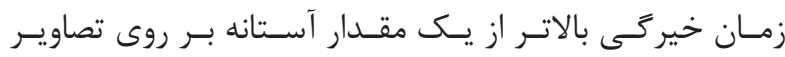

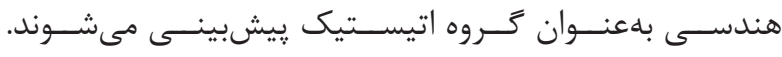

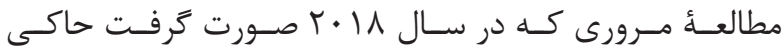

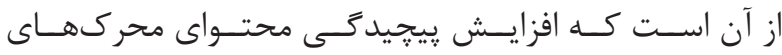

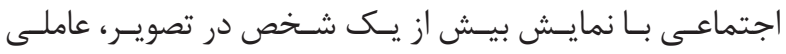

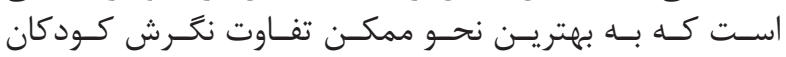

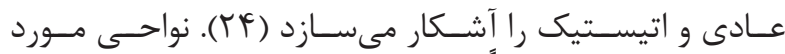

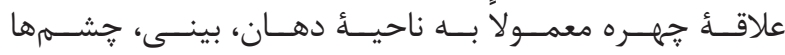

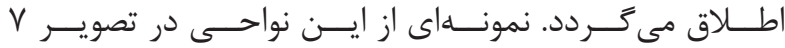

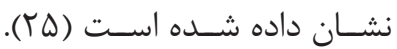

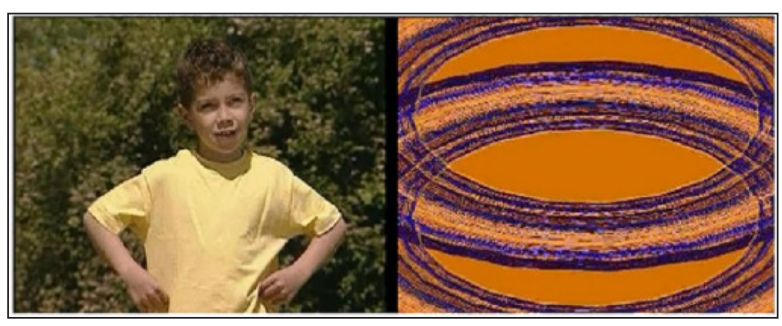

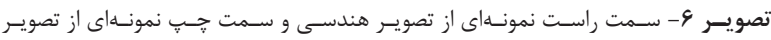

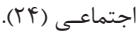

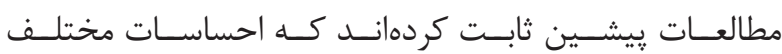

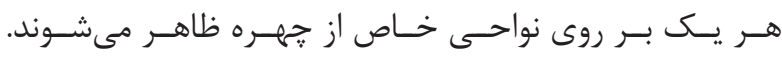

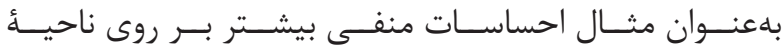

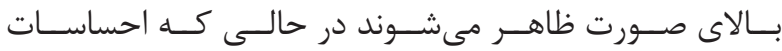




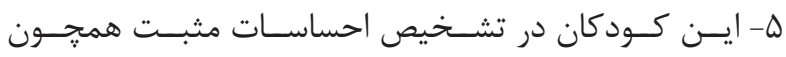

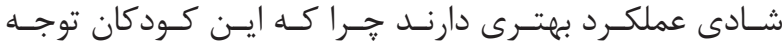

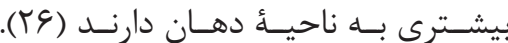

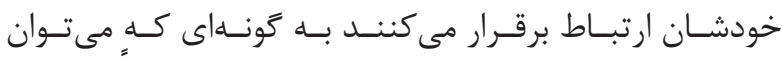

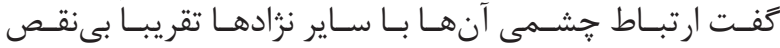

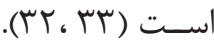

جدول ا- مشخصات كلى مطالعات صورت گرفته.

\begin{tabular}{|c|c|c|c|c|c|c|c|c|}
\hline \multirow{2}{*}{ رويكرث يادكيرى } & \multirow{2}{*}{ معرى } & \multirow{2}{*}{ وظليفه } & \multicolumn{2}{|c|}{ تعداد } & \multirow{2}{*}{ رويكرد تحليل } & \multirow{2}{*}{ نوع مطالعه } & \multirow{2}{*}{ سال } & \multirow{2}{*}{ عنيك } \\
\hline & & & TD & ASD & & & & \\
\hline \multirow[t]{2}{*}{ 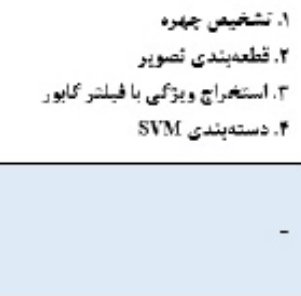 } & نصوير & 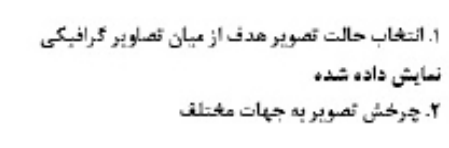 & $A$ & $\wedge$ & 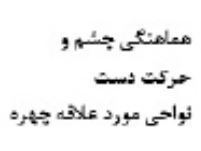 & نحليل & $r+11$ & $(\mathrm{TA})$ \\
\hline & تصوينو & 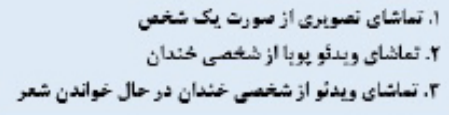 & & & 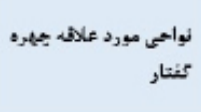 & نحلبل & $r+1 r$ & (r4) \\
\hline - & تصوير & 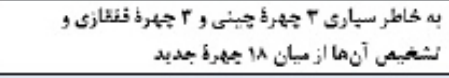 & $\Delta \Lambda$ & $T 9$ & 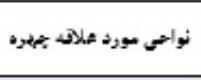 & نمليل & $r-19$ & (Tr) \\
\hline 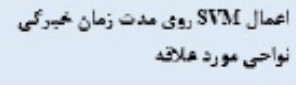 & ويدنو & 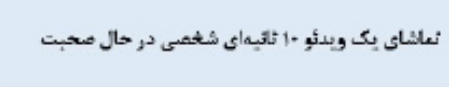 & rv & TV & 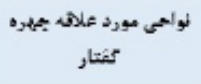 & نشخيص ن ن نمليل & $r+11$ & $(T \Delta)$ \\
\hline \multirow[t]{2}{*}{ 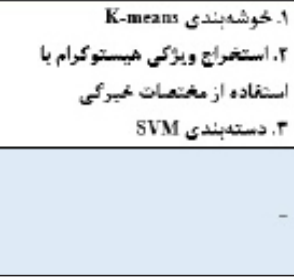 } & نصوير & 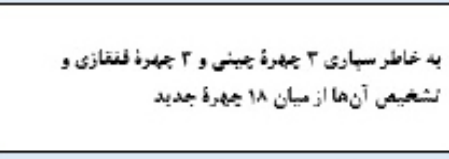 & $\Delta A$ & rq & 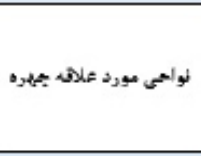 & نمبيد & $r-19$ & (TT) \\
\hline & تصوير & 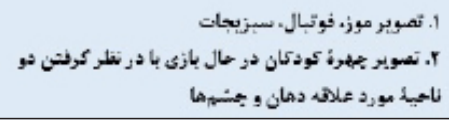 & ri & $r$ & نواحى بورد علالته جيروه & نحليل & $r+11$ & (1) \\
\hline 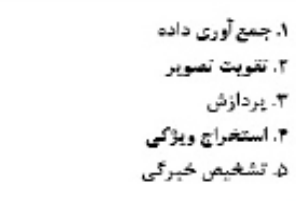 & ويدئو & 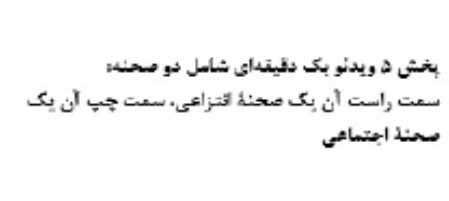 & rT & $\wedge$ & ثرجيح عندسى & تشخيص & $r+1 \mathrm{~V}$ & $\langle\mathrm{r}-\rangle$ \\
\hline 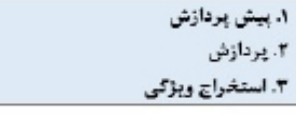 & ويدئو & 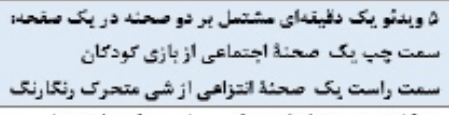 & $r$ & $\wedge$ & ترجبح هندسى & نحلبل & $r-18$ & $\langle\mathrm{r} \mid\rangle$ \\
\hline - & & 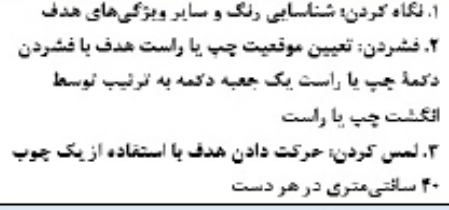 & $1 \%$ & if & 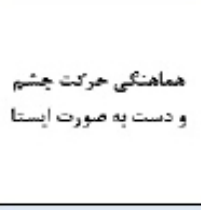 & نمليل & $t+14$ & (Tr) \\
\hline - & ويدئو & 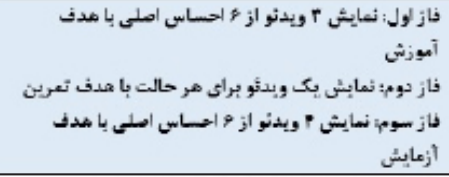 & is & $T$ & تشتشبص احساسات & نحلبل & $r+1 *$ & (rs) \\
\hline 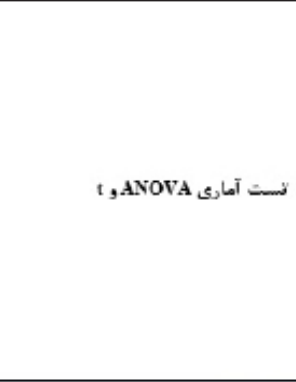 & ويدنو & 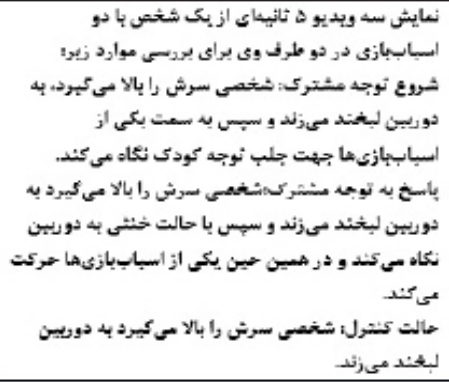 & $\cdot$ & 11 & تيوبه هشتِرك & تمليل و ترعان & $r+1 v$ & $(T 0)$ \\
\hline 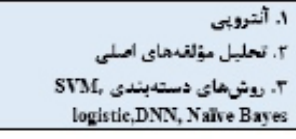 & ويدئو & نعاشاى rا ويدئو +r ثائيهاى & Th & TF & توجه مشترى & تشبعبص & $t+14$ & (TV) \\
\hline 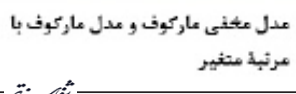 & ويدئو & هاثرش يك ربدئو r دفيدماى از نعاشاى نوزاد به صورت & rs & 3 & نواحى جيثرث & تشبكب & $r+11$ & (Ts) \\
\hline
\end{tabular}


جدول r- نوآورى و نتايج مطالعات صورت كرفته.

\begin{tabular}{|c|c|c|}
\hline تكايع & نولَّكا & عبي \\
\hline 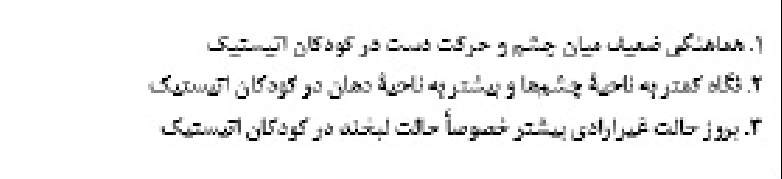 & 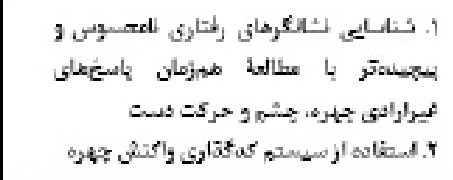 & $(\% N)$ \\
\hline 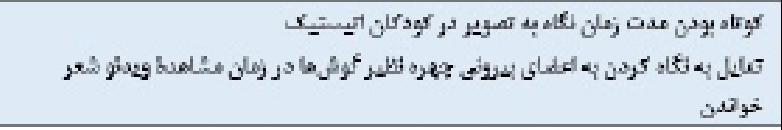 & 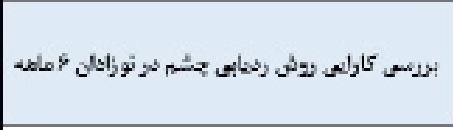 & (ะข) \\
\hline 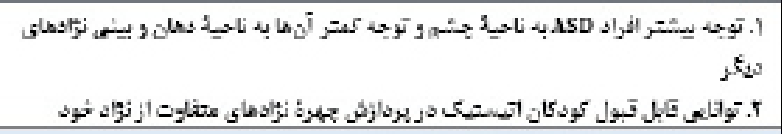 & 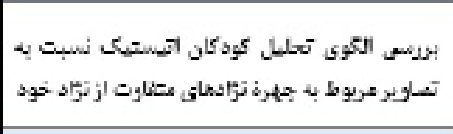 & $(\pi)$ \\
\hline 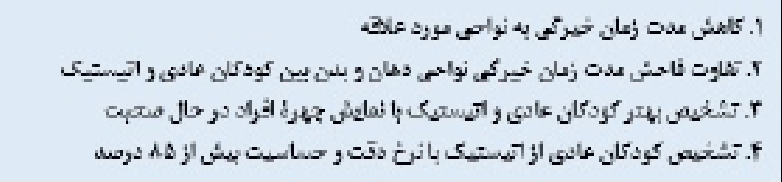 & 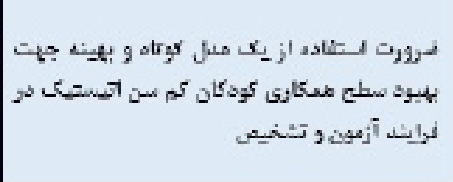 & $(\% \Delta)$ \\
\hline 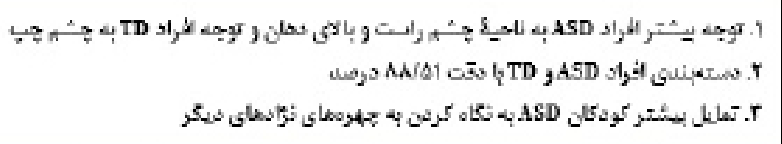 & 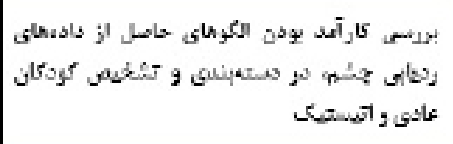 & (rr) \\
\hline 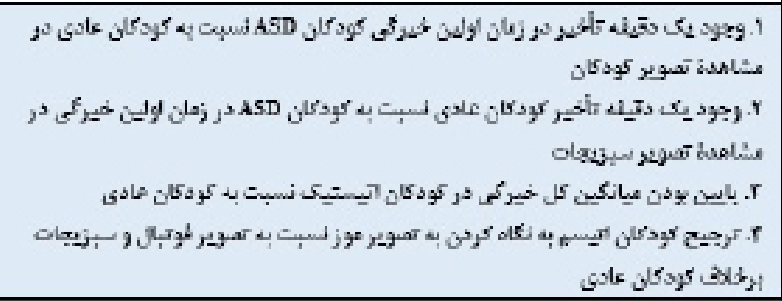 & 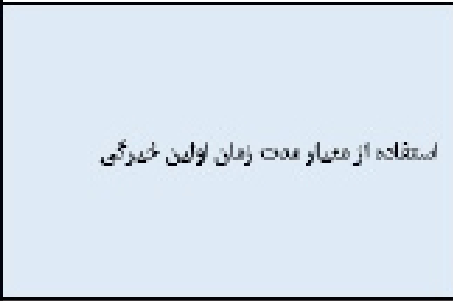 & (1) \\
\hline 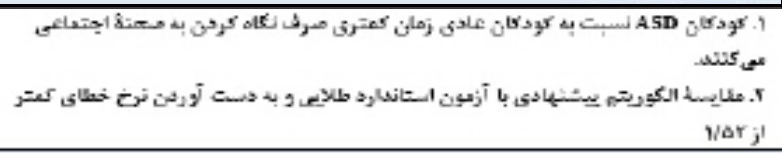 & 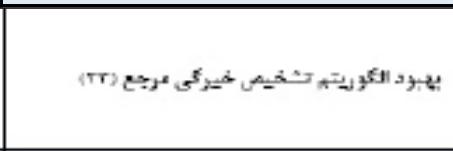 & $(t *)$ \\
\hline 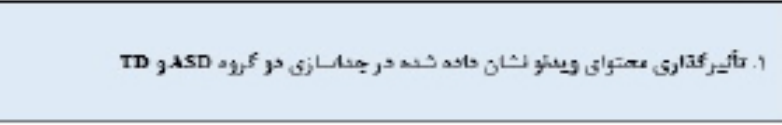 & 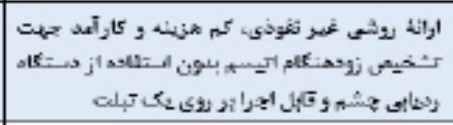 & (r) \\
\hline 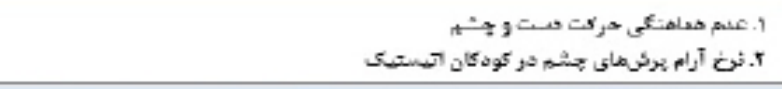 & الستاده از ععيار يرش & (TT) \\
\hline 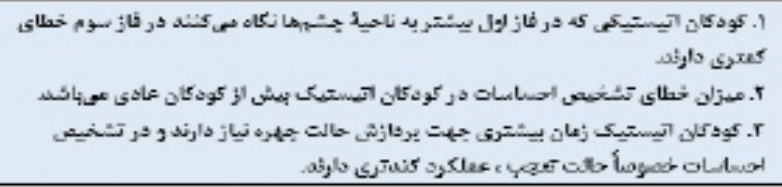 & 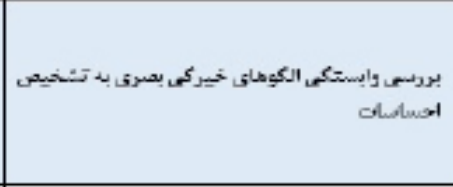 & $\langle\Gamma \varphi\rangle$ \\
\hline 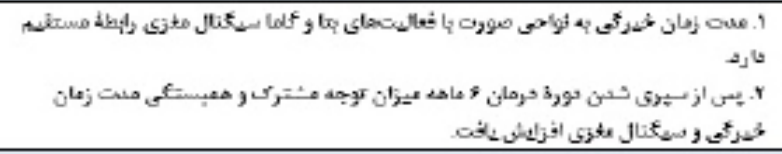 & 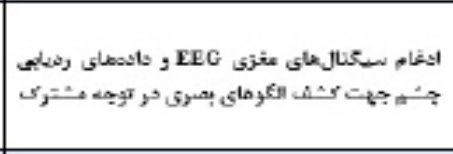 & (TSI \\
\hline 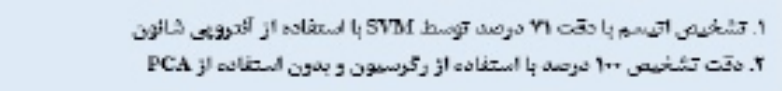 & 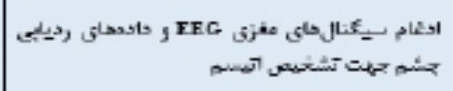 & (TV) \\
\hline 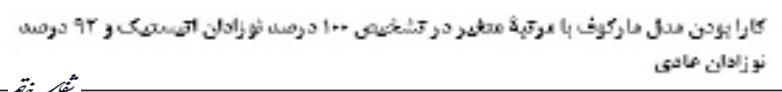 & 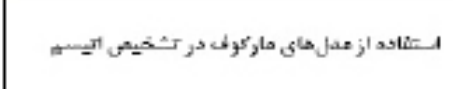 & $(T+)$ \\
\hline
\end{tabular}

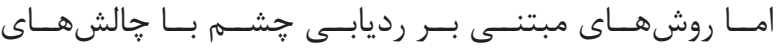

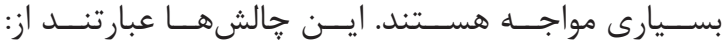

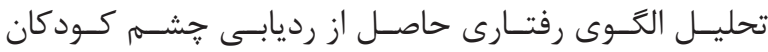

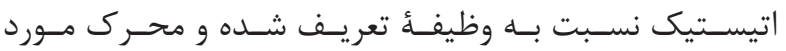

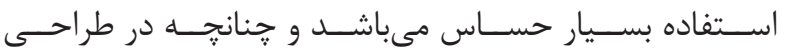

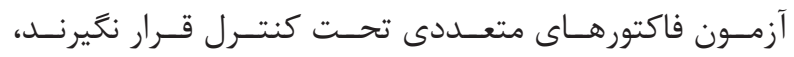

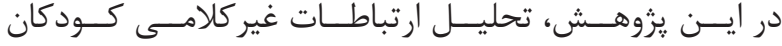

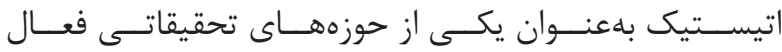

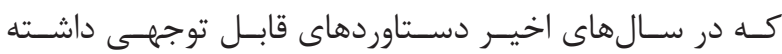

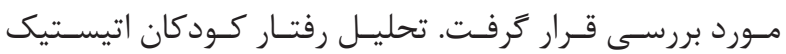

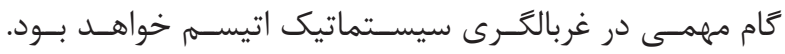




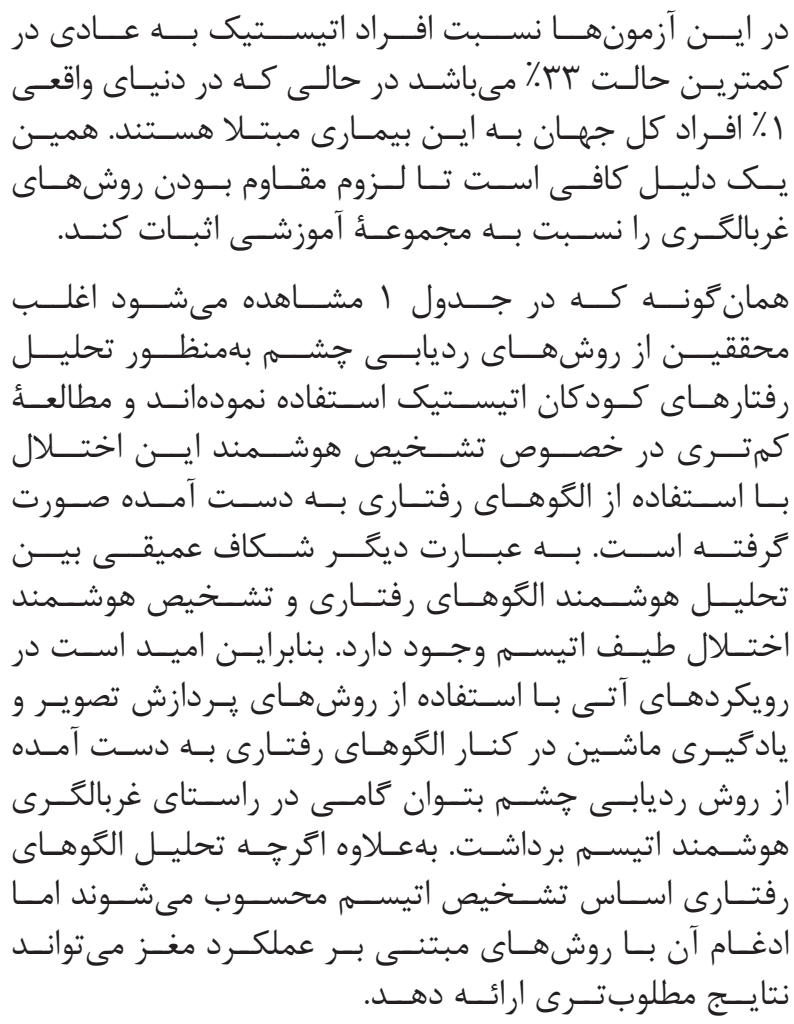

1. Almourad MB, Bataineh E, Stocker J, Marir F. Analyzing the behavior of autistic and normal developing children using eye tracking data. International Conference on Kansei Engineering \& Emotion Research. 2018; 340-9.

2. Lai MC, Lombardo MV, Baron-Cohen S. Autism. The Lancet. 2014; 383(9920): 896-910.

3. Akbari Bayatiani Z. Autism spectrum disorder from diagnosis to treatment. Shefaye Khatam. 2018; 6(4): 93-101.

4. Bozgeyikli L, Raij A, Katkoori S, Alqasemi R. A survey on virtual reality for individuals with autism spectrum disorder: design considerations. IEEE Transactions on Learning Technologies. 2018; 11(2): 133-51.

5. Sarrett JC, Rommelfanger KS. Commentary: attention to eyes is present but in decline in 2-6-month-old infants later diagnosed with autism. Front Public Health. 2015; 3: 272. doi: 10.3389/fpubh.2015.00272.

6. Dolan WN. Using the autism diagnostic observation schedule (ADOS) to discriminate between children with autism and children with language impairments without autism. Master Thesis. Louisiana State University and Agricultural and Mechanical College. 2009.

7. Wang J, Wang Q, Zhang H, Chen J, Wang S, Shen D. Sparse multiview task-centralized ensemble learning for ASD diagnosis based on age-and sex-related functional

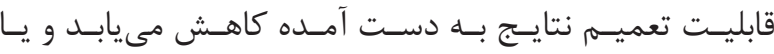

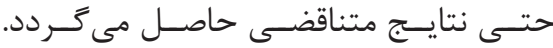

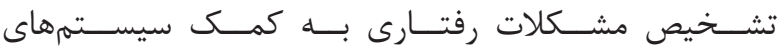

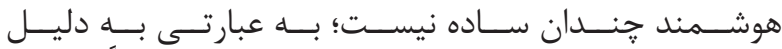

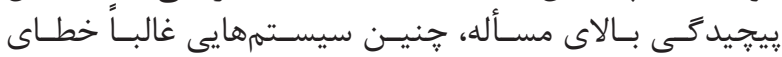

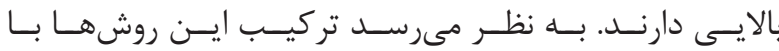

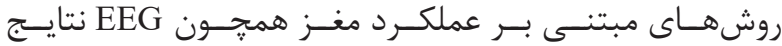

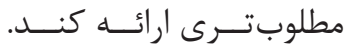

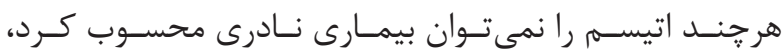

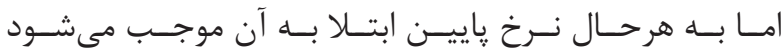

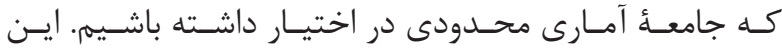

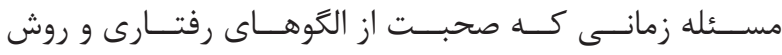

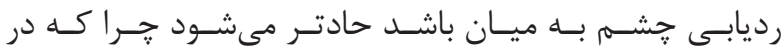

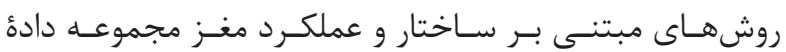

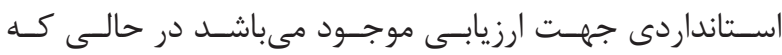

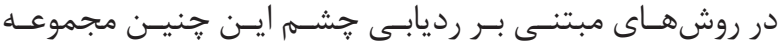

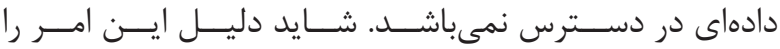

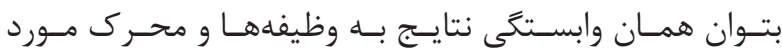

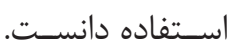

$$
\begin{aligned}
& \text { منابع }
\end{aligned}
$$

connectivity patterns. IEEE Transactions on Cybernetics. 2018; 49(8): 3141-54.

8. Tejwani R, Liska A, You H, Reinen J, Das P. Autism classification using brain functional connectivity dynamics and machine learning. arXiv preprint arXiv:171208041. 2017.

9. Gök M. A novel machine learning model to predict autism spectrum disorders risk gene. Neural Computing and Applications. 2018; 5: 1-7.

10. Cogill S, Wang L. Support vector machine model of developmental brain gene expression data for prioritization of Autism risk gene candidates. Bioinformatics. 2016; 32(23): 3611-8.

11. Cicarelli Silva A, Varanda C. Eye-tracking technique as an instrument in the diagnosis of autism spectrum disorder. Autism Journal of Autism \& Related Disabilities. 2017; 3(3).

12. Frazier TW, Klingemier EW, Parikh S, Speer L, Strauss MS, Eng C, et al. Development and validation of objective and quantitative eye tracking-based measures of autism risk and symptom levels. J Am Acad Child Adolesc Psychiatry. 2018; 57(11): 858-66.

13. Frazier TW, Klingemier EW, Parikh S, Speer L, Strauss MS, Eng C, et al. Development and Validation of objective and quantitative eye tracking- based measures of autism risk and symptom levels. Journal of the 
American Academy of Child \& Adolescent Psychiatry. 2018; 57(11): 858-66.

14. Murias M, Major S, Davlantis K, Franz L, Harris A, Rardin B, et al. Validation of eye-tracking measures of social attention as a potential biomarker for autism clinical trials. Autism Res. 2018; 11(1): 166-74.

15. Nagel GL. Use of eye tracking for esports analytics in a MOBA game, Master Thesis. The University of Bergen, Faculty of Social Sciences. 2017.

16. Gredebäck G, Johnson S, Von Hofsten C. Eye tracking in infancy research. Dev Neuropsychol. 2009; 35(1): 1-19.

17. Lund H. Eye tracking in library and information science: a literature review. Library Hi Tech. 2016; 34(4): 585-614.

18. Nystrom M, Holmqvist K. An adaptive algorithm for fixation, saccade, and glissade detection in eyetracking data. Behav Res Methods. 2010; 42(1): 188-204.

19. Liversedge S, Gilchrist I, Everling S. The Oxford handbook of eye movements: Oxford University Press. 2011.

20. Liversedge SP, Findlay JM. Saccadic eye movements and cognition. Trends Cogn Sci. 2000; 4(1): 6-14.

21. Boucheix J-M, Lowe RK. An eye tracking comparison of external pointing cues and internal continuous cues in learning with complex animations. Learning and Instruction. 2010; 20(2): 123-35.

22. Mahmoody M, Gobari Bonab B, Shokoohi Yekta M, Pooretemad HR, Akbari Zardkhaneh S. A preliminery study on the development of an expert system for diagnosing 2-6 years old autisitic children. Journal of Psychology. 2014; 18: 94-110.

23. Shihab I. Classification and monitoring of autism using SVM and VMCM. Journal of Theoretical and Applied Information Technology. 2018; 96(14):4379-89.

24. Moore A, Wozniak M, Yousef A, Barnes CC, Cha D, Courchesne E, et al. The geometric preference subtype in ASD: identifying a consistent, early-emerging phenomenon through eye tracking. Mol Autism. 2018; 9(1): 19-32.

25. Wan G, Kong X, Sun B, Yu S, Tu Y, Park J, et al. Applying eye tracking to identify autism spectrum disorder in children. J Autism Dev Disord. 2018; 49(1): 209-15.

26. Bal E, Harden E, Lamb D, Van Hecke AV, Denver
JW, Porges SW. Emotion recognition in children with autism spectrum disorders: relations to eye gaze and autonomic state. J Autism Dev Disord. 2010; 40(3): 358-70.

27. Thapaliya S, Jayarathna S, Jaime M. Evaluating the EEG and eye movements for Autism spectrum disorder. 2018 IEEE international conference on big data (Big Data). 2018; 2328-36.

28. Samad MD, Diawara N, Bobzien JL, Harrington JW, Witherow MA, Iftekharuddin KM. A feasibility study of autism behavioral markers in spontaneous facial, visual, and hand movement response data. IEEE Trans Neural Syst Rehabil Eng. 2018; 26(2): 353-61.

29. Shic F, Macari S, Chawarska K. Speech disturbs face scanning in 6-month-old infants who develop autism spectrum disorder. Biol Psychiatry. 2014; 75(3): 231-7.

30. Vargas-Cuentas NI, Roman-Gonzalez A, Gilman RH, Barrientos F, Ting J, Hidalgo D, et al. Developing an eye-tracking algorithm as a potential tool for early diagnosis of autism spectrum disorder in children. PLoS One. 2017 ; 12(11): e0188826.

31. Vargas-Cuentas NI, Hidalgo D, Roman-Gonzalez A, Power M, Gilman RH, Zimic M. Diagnosis of autism using an eye tracking system. 2016 IEEE Global Humanitarian Technology Conference (GHTC); Seattle, WA2016. 624-7.

32. Yi L, Quinn PC, Fan Y, Huang D, Feng C, Joseph L, et al. Children with Autism spectrum disorder scan ownrace faces differently from other-race faces. J Exp Child Psychol; 2016; 141: 177-86.

33. Liu W, Li M, Yi L. Identifying children with autism spectrum disorder based on their face processing abnormality: A machine learning framework. Autism Res. 2016; 9(8): 888-98.

34. Crippa A, Forti S, Perego P, Molteni M. Eye-hand coordination in children with high functioning autism and Asperger's disorder using a gap-overlap paradigm. J Autism Dev Disord. 2013; 43(4): 841-50.

35. Billeci L, Narzisi A, Tonacci A, Sbriscia-Fioretti B, Serasini L, Fulceri F, et al. An integrated EEG and eye-tracking approach for the study of responding and initiating joint attention in Autism spectrum disorders. Sci Rep. 2017; 7(1): 13560. doi: 10.1038/s41598-01713053-4.

36. Alie D, Mahoor MH, Mattson WI, Anderson DR, Messinger DS. Analysis of eye gaze pattern of infants at risk of autism spectrum disorder using markov models. 2011 IEEE Workshop on Applications of Computer Vision (WACV). 2011; 282-7. 